\title{
NONNEGATIVE RECTANGULAR MATRICES HAVING CERTAIN NONNEGATIVE $W$-WEIGHTED GROUP INVERSES
}

\author{
S. K. JAIN
}

\begin{abstract}
Nonnegative rectangular matrices having nonnegative $W$-weighted group inverses are characterized. Our techniques suggest an interesting approach to extend the earlier known results on $\lambda$-monotone square matrices to rectangular ones. We also answer a question of characterizing nonnegative matrices having a nonnegative solution $X$ where (1) $A=A X A$, (2) $X=X A X$, (3) $(A X)$ is 0 -symmetric, (4) $(X A)$ is 0 -symmetric. In particular, we obtain theorems of Berman-Plemmons and Plemmons-Cline characterizing nonnegative matrices $A$ with a nonnegative MoorePenrose inverse. Matrices having nonnegative generalized inverses are of interest in the study of finding nonnegative best approximate solutions of linear systems. Such matrices are of considerable interest in statistics, numerical linear algebra and mathematical economics.
\end{abstract}

1. Introduction. Let $A$ be an $m \times n$ real matrix. Consider the equations: (1) $A X A=A$, (2) $X A X=X$, (3) $(A X)^{T}=A X$, (4) $(X A)^{T}=X A$ and (5) $A X=X A$ where $X$ is an $n \times m$ real matrix and " $T$ " denotes the transpose. For a rectangular matrix $A$ and for a nonempty subset $\lambda$ of $\{1,2,3,4,5\}, X$ is called a $\lambda$-inverse of $A$ if $X$ satisfies equation (i) for each $i \in \lambda$. In particular, the $\{1,2,3,4\}$-inverse of $A$ is the unique Moore-Penrose generalized inverse and is denoted by $A^{\dagger}$. A $\{1,2\}$-inverse of $A$ which satisfies (5) is necessarily square and is called a group inverse. The group inverse of $A$, if it exists, is unique and is denoted by $A^{\#}$. A matrix $A$ is called $\lambda$-monotone if a $\lambda$-inverse exists and is nonnegative. Let $A, W$ be respectively $m \times n$, $n \times m$ matrices, such that $(A W)^{\#}$ exists. Consider the equations $(1)_{w}(A W)=$ $(A W)^{2} X W,(2)_{w} X=X W A W X$, and (5) ${ }_{w} A W X=X W A$. Following Cline-Greville [3], $X\left((A W)^{\#}\right)^{2} A$ is the unique solution of $(1)_{w},(2)_{w}$ and $(5)_{w}$ and is called the $W$-weighted group inverse of $A$.

A matrix $A=\left(a_{i j}\right)$ is called 0 -symmetric if $a_{i j}=0$ implies $a_{j i}=0$. A square matrix is called weakly 0 -symmetric if the $i$ th row of $A$ is zero if and only if the $i$ th row of $A^{T}$ is zero. For a square matrix $A$, the smallest positive integer $k$ such that rank $A^{k}=\operatorname{rank} A^{k+1}$ is the index of $A$. If a matrix is the direct sum of matrices $S_{i}$, then $S_{i}$ are called summands of $A$. Throughout $S_{n}$ will denote the symmetric group on $n$ objects.

Let $A, W$ be nonnegative matrices of sizes $m \times n, n \times m$ respectively such that the $i$ th row (column) of $W$ is zero if and only if the $i$ th column (row) of $A$ is zero and

Received by the editors July 18, 1980; presented to the Society, January 1980 at San Antonio, Texas. 1980 Mathematics Subject Classification. Primary 15A09, 15A48.

Key words and phrases. Nonnegative matrices, $W$-weighted group inverse.

(C)1982 American Mathematical Society $0002-9939 / 81 / 0000-0053 / \$ 02.50$ 
$\operatorname{rank} A=\operatorname{rank} A W$. Theorem 2 of this paper characterizes all nonnegative $W$ weighted group inverses of $A$. By choosing $W=A^{\dagger}$, we obtain the theorem of Plemmons-Cline regarding the structure of nonnegative matrices $A$ with $A^{\dagger} \geqslant 0$. The other choices of $W$ are also discussed in the remarks. The corollary to Theorem 1 characterizes all nonnegative matrices $X$ such that (1) $A X A=A$, (2) $X A X=X,(3)_{0}$ ( $A X)$ is weakly 0 -symmetric, (4) $X A$ is weakly 0 -symmetric, where $A$ is a nonnegative $m \times n$ matrix. In particular, we rederive the theorem of Plemmons-Cline that if $A \geqslant 0$ then $A^{\dagger} \geqslant 0$ if and only if $A^{\dagger}=D A^{T}$ where $D$ is a diagonal matrix with all entries on the diagonal as positive. In our investigations one of the key results is to obtain nonnegative solutions $X, Y$ (not necessarily square) of simultaneous matrix equations

$$
X Y=\operatorname{diag}\left(X_{1}, \ldots, X_{r}, 0, \ldots, 0\right), \quad Y X=\operatorname{diag}\left(Y_{1}, \ldots, Y_{s}, 0, \ldots, 0\right)
$$

where $X_{i}$ and $Y_{i}$ are square matrices of orders $a_{i}$ and $\alpha_{i}$ respectively (Lemmas 2 and 3). The proof is similar to the proof in [6, Lemmas 1 and 2] but needs somewhat careful computations and the suitable partitionings of $X$ and $Y$ since, in this case, $a_{i}$, $\alpha_{i}$ are not necessarily equal and the number of positive blocks on the diagonals of $X Y$ and $Y X$ are not given to be equal, although we do show that in order that there is a solution, $r$ must be equal to $s$.

We shall need the following lemmas proved in [5].

Lemma A [5, LemMa 2]. Let $A, C_{1}, \ldots, C_{n}$ be nonnegative rectangular matrices of suitable sizes such that $A C_{i}=0\left(C_{i} A=0\right), i=1, \ldots, n$, and

$$
X A+\sum_{i=1}^{n} C_{i} Y_{i}>0 \quad\left(A X+\sum_{i=1}^{n} Y_{i} C_{i}>0\right)
$$

for some nonnegative matrices $X_{i}, Y_{i}$ of suitable sizes, $1 \leqslant i \leqslant n$. Then $A=0$ or all $C_{i}=0$.

2. Preliminary results. We begin with a statement of a simple but frequently used fact contained in

SUblemMA 1. Let $A=\left(a_{i j}\right)$ be an $m \times n$ nonnegative matrix and let $B$ be an $n \times m$ matrix, such that the ith row of the matrix $A B$ is zero. Then either the ith row of $A$ is zero or the jth row of $B$ is zero where $a_{i j} \neq 0$. There is a similar result for the columns.

LEMMA 1. Let $A$ be a nonnegative $m \times n$ matrix and $W$ be a nonnegative $n \times m$ matrix such that the ith row (column) of $W$ is nonzero if and only if the ith column (row) of $A$ is nonzero. Then $A W$ and $W A$ are weakly 0 -symmetric. Further, if $B$ is $a$ nonnegative 0-symmetric square matrix having $B^{\#} \geqslant 0$, then $B B^{\#}$ is also 0-symmetric.

Proof. The proof is a straightforward application of Sublemma 1.

LEMMA 2. Let $X, Y$ be respectively $m \times n, n \times m$ nonnegative matrices such that

$$
X Y=\left[\begin{array}{ccc}
X_{1} & & 0 \\
& \ddots & \\
0 & & X_{r}
\end{array}\right] \text { and } Y X=\left[\begin{array}{ccc}
Y_{1} & & 0 \\
& \ddots & \\
0 & & Y_{s}
\end{array}\right] \text {, }
$$


where $X_{i}, Y_{i}$ are positive square matrices of orders $a_{i}, \alpha_{i}$ respectively. Then

(i) $r=s$; and

(ii) if $X=\left(X_{i j}\right), Y=\left(Y_{i j}\right)$ are partitionings of $X, Y$ respectively, such that $X_{i i}, Y_{i i}$ are of orders $a_{i} x \alpha_{i}, \alpha_{i} x a_{i}$ respectively, then $X_{j \sigma(j)} \neq 0, Y_{\sigma(j) j} \neq 0, X_{j k}=0=Y_{k j}$ for all $k \neq \sigma(j)$ for some $\sigma \in S_{r}$.

Proof. Let $r>s$. Write

$$
X_{s}^{\prime}=\left[\begin{array}{ccc}
X_{s} & & \\
& \ddots & \\
& & X_{r}
\end{array}\right] \text { and } a_{s}^{\prime}=\sum_{i=s}^{r} a_{i} .
$$

Then $X_{s}^{\prime}$ is of order $a_{s}^{\prime}$ and has zero entries. Let $X=\left(X_{i j}\right), Y=\left(Y_{i j}\right)$ be the partitioning of $X$ and $Y$ such that $X_{i i}, Y_{i i}$ are of orders respectively $a_{i} x \alpha_{i}, \alpha_{i} x a_{i}$, $1 \leqslant i \leqslant s-1 . X_{s s}, Y_{s s}$ are of orders $a_{s}^{\prime} x \alpha_{s}$ and $\alpha_{s} x a_{s}^{\prime}$ respectively. Then $X_{j}=$ $\sum_{k=1}^{s} X_{j k} Y_{k j}>0,1 \leqslant j \leqslant s-1, X_{i t} Y_{t k}=0, i \neq k, 1 \leqslant t \leqslant s$. Using Lemma A, we obtain that, for each $j \in\{1, \ldots, s-1\}$, there exists an integer $l \in\{1, \ldots, r\}$ such that $X_{j}=X_{j l} Y_{l j}$, and $X_{j k}=0=Y_{k j} \forall k \neq l$. Thus, there is one and only one nonzero block in each but the $s$ th row of $X$ and in each but the $s$ th column of $Y$. Considering

$$
Y X=\left[\begin{array}{ccc}
Y_{1} & & 0 \\
& \ddots & \\
0 & & Y_{s}
\end{array}\right],
$$

we obtain that there is one and only one nonzero block in each column of $X$ and in each row of $Y$. Thus $X$ has exactly $s$ nonzero blocks, which implies that the $s$ th row of $X$ will also have exactly one nonzero block. Similarly, the $s$ th column of $Y$ will have exactly one nonzero block. This gives a permutation $\sigma \in S_{r}$, the symmetric group on $r$ symbols $\{1, \ldots, r\}$ such that $X_{j \sigma(j)} \neq 0, Y_{\sigma(j) j} \neq 0$ and $X_{j k}=0=Y_{k j}$ for all $k \neq \sigma(j)$. Clearly,

$$
X_{i \sigma(i)} Y_{\sigma(i) i}=X_{i}, \quad 1 \leqslant i \leqslant s-1, \quad X_{s \sigma(s)} Y_{\sigma(s) s}=X_{s}^{\prime}
$$

Also,

$$
Y_{\sigma(i) i} X_{i \sigma(i)}=Y_{\sigma(i)}, \quad 1 \leqslant i \leqslant s .
$$

From (1) we have

$$
X_{s \sigma(s)} Y_{\sigma(s) s}=X_{s}^{\prime}=\left[\begin{array}{lll}
X_{s} & & 0 \\
& \ddots & \\
0 & & X_{r}
\end{array}\right]
$$

and so

$$
\left(X_{s \sigma(s)} Y_{\sigma(s) s}\right)\left(X_{s \sigma(s)} Y_{\sigma(s) s}\right)=\left[\begin{array}{lll}
X_{s}^{2} & & 0 \\
& \ddots & \\
0 & & X_{r}^{2}
\end{array}\right]
$$


But since $Y_{\sigma(s) s} X_{s \sigma(s)}>0$, and $X_{s \sigma(s)}, Y_{\sigma(s) s}$ cannot have zero row, zero column respectively (by equation (3)), we obtain

$$
X_{s \sigma(s)}\left(Y_{\sigma(s) s} X_{s \sigma(s)}\right) Y_{\sigma(s) s}>0 .
$$

But (5) contradicts (4).

Hence $r=s$, proving (i). (ii) follows from the proof of (i) given above.

LEMMA 3. Let $X, Y$ be nonnegative matrices of orders $m \times n, n \times m$ respectively, such that $X Y=\left(\begin{array}{ll}A & 0 \\ 0 & 0\end{array}\right), Y X=\left(\begin{array}{ll}B & 0 \\ 0 & 0\end{array}\right)$ where $A, B$ are square matrices of orders $a$ and $b$ respectively and $\operatorname{diag} A>0, \operatorname{diag} B>0$. If

$$
X=\left[\begin{array}{ll}
X_{1} & X_{2} \\
X_{3} & X_{4}
\end{array}\right] \text { and } Y=\left[\begin{array}{ll}
Y_{1} & Y_{2} \\
Y_{3} & Y_{4}
\end{array}\right]
$$

are partitioned, such that $X_{1}, X_{4}, Y_{1}, Y_{4}$ are of orders $a \times \alpha,(m-a) \times(n-\alpha)$, $\alpha \times a,(n-\alpha) \times(m-a)$ respectively, then

$$
X=\left[\begin{array}{ll}
X_{1} & 0 \\
0 & X_{4}
\end{array}\right] \text { and } Y=\left[\begin{array}{ll}
Y_{1} & 0 \\
0 & Y_{4}
\end{array}\right]
$$

where $X_{1} Y_{1}=A, Y_{1} X_{1}=B$, and $X_{4} Y_{4}=0=Y_{4} X_{4}$.

Proof. The proof is straightforward.

The following is another fact.

Sublemma 2. Let $X, Y$ be respectively $m \times n, n \times m$ matrices such that (i) rank $X Y=\operatorname{rank} X$, (ii) index $X Y=1$. Then $\operatorname{rank} X Y=\operatorname{rank} Y X$ and index $Y X=1$. Further if $X \geqslant 0, Y \geqslant 0$, then $(X Y)^{\#} \geqslant 0$ if and only if $(Y X)^{\#} \geqslant 0$.

\section{Main results.}

THEOREM 1. Let $A$ be a nonnegative $m \times n$ matrix and $W$ be a nonnegative $n \times m$ matrix such that the ith row (column) of $W$ is nonzero if and only if the ith column (row) of $A$ is nonzero and $\operatorname{rank} A W=\operatorname{rank} A$. Then $(A W)^{\#} \geqslant 0$ if and only if there exists permutation matrices $P$ and $Q$ of orders $m$ and $n$ respectively such that $P A Q^{T}$ is a direct sum of matrices of the following types (not necessarily all):

(a) $x y^{T}$ where $x, y$ are positive vectors of suitable sizes (not necessarily of the same size);

(b)

$$
\left[\begin{array}{ccccc}
0 & x_{1} y_{2}^{T} & 0 & \ldots & 0 \\
0 & 0 & x_{2} y_{3}^{T} & \ldots & 0 \\
x_{d} y_{1}^{T} & 0 & 0 & \ldots & 0
\end{array}\right]
$$

where $x_{i}, y_{i}$ are positive vectors of suitable sizes (not necessarily of the same size);

(c) zero matrix (not necessarily square),

and $Q W(A W)^{\#} P^{T}$ is a direct sum of matrices of the following types (not necessarily all);

( $\left.\mathrm{a}^{\prime}\right) u v^{T}$, where $u, v$ are positive vectors of suitable sizes; 
$\left(b^{\prime}\right)$

$$
\left[\begin{array}{ccccc}
0 & 0 & 0 & \ldots & u_{d} v_{1}^{T} \\
u_{1} v_{2}^{T} & 0 & 0 & \ldots & 0 \\
0 & u_{2} v_{3}^{T} & 0 & \ldots & 0 \\
\vdots & & & & \vdots \\
0 & 0 & 0 & u_{d-1} v_{d}^{T} & 0
\end{array}\right]
$$

where $u_{i}, v_{i}$ are positive vectors of suitable sizes;

$\left(c^{\prime}\right)$ zero matrix of suitable size.

Proof. We first show rank $A W=\operatorname{rank} W(A W)^{\#}$. Now rank $A W=\operatorname{rank}(A W)^{\#}$ $=\operatorname{rank}(A W)(A W)^{\#} \leqslant \operatorname{rank} W(A W)^{\#} \leqslant \operatorname{rank}(A W)^{\#}=\operatorname{rank} A W$, proving the claim.

Set $X=A, Y=W(A W)^{\#}$. Then $X Y=(A W)(A W)^{\#}$ is a nonnegative weakly 0 -symmetric idempotent (Lemma 1). Also, $Y X=W(A W)^{\#} A=(W A)^{\#} W A$ is a weakly 0 -symmetric idempotent (Lemma 1 and Sublemma 2). Therefore by [4, Theorem 2], there exist permutation matrices $P_{1}, Q_{1}$ of orders $m, n$ respectively such that

$$
P_{1} X Y P_{1}^{T}=\left[\begin{array}{llll}
g_{1} h_{1}^{T} & & & 0 \\
& \ddots & & \\
& & g_{r} h_{r}^{T} & \\
0 & & & 0
\end{array}\right] \text { and } Q_{1} Y X Q_{1}^{T}=\left[\begin{array}{llll}
p_{1} q_{1}^{T} & & & 0 \\
& \ddots & & \\
& & p_{s} q_{2}^{T} & \\
0 & & & 0
\end{array}\right]
$$

where $g_{i}, h_{i}\left(p_{i}, q_{i}\right)$ are positive vectors of the same order, say $a_{i}\left(b_{i}\right)$ and $h_{i}^{T} g_{i}=1$ $\left(q_{i}^{T} p_{i}=1\right)$ and $\operatorname{rank} X Y=r, \operatorname{rank} Y X=s$. Let $L=P_{1} X Q_{1}^{T}, M=Q_{1} Y P_{1}^{T}$.

$$
C=\left[\begin{array}{ccc}
g_{1} h_{1}^{T} & & \\
& \ddots & \\
& & g_{r} h_{r}^{T}
\end{array}\right] \text { and } D=\left[\begin{array}{lll}
p_{1} q_{1}^{T} & & \\
& \ddots & \\
& & p_{s} q_{s}^{T}
\end{array}\right]
$$

Then

$$
L M=\left[\begin{array}{ll}
C & 0 \\
0 & 0
\end{array}\right] \text { and } M L=\left[\begin{array}{ll}
D & 0 \\
0 & 0
\end{array}\right]
$$

where $\operatorname{diag} C>0, \operatorname{diag} D>0$. By Lemma 3,

$$
L=\left[\begin{array}{ll}
L^{\prime} & 0 \\
0 & L^{\prime \prime}
\end{array}\right] \text { and } M=\left[\begin{array}{ll}
M^{\prime} & 0 \\
0 & M^{\prime \prime}
\end{array}\right]
$$

where

$$
L^{\prime} M^{\prime}=C=\left[\begin{array}{ccc}
g_{1} h_{1}^{T} & & \\
& \ddots & \\
& & g_{r} h_{r}^{T}
\end{array}\right] \text { and } M^{\prime} L^{\prime}=D=\left[\begin{array}{ccc}
p_{1} q_{1}^{T} & & \\
& \ddots & \\
& & p_{s} q_{s}^{T}
\end{array}\right]
$$


By Lemma 1 (or by Sublemma 2), $r=s$. Also by Lemma 1 , if

$$
L^{\prime}=\left[\begin{array}{lll}
L_{11}^{\prime} & \ldots & L_{1 r}^{\prime} \\
\vdots & & \\
L_{r 1}^{\prime} & \ldots & L_{r r}^{\prime}
\end{array}\right] \text { and } M^{\prime}=\left[\begin{array}{lll}
M_{11}^{\prime} & \ldots & M_{1 r}^{\prime} \\
\vdots & & \\
M_{r 1}^{\prime} & \ldots & M_{r r}^{\prime}
\end{array}\right]
$$

are partitionings of $L^{\prime}, M^{\prime}$ such that $L_{i i}^{\prime}, M_{i i}^{\prime}$ are of orders $a_{i} \times \alpha_{i}, \alpha_{i} \times a_{i}$ respectively, where $g_{i} h_{i}^{T}$ and $p_{i} q_{i}^{T}$ respectively are of orders $a_{i}$ and $\alpha_{i}$, then

$$
\begin{aligned}
L_{i \sigma(i)}^{\prime} \neq 0, & L_{i j}^{\prime}=0 \quad \forall j \neq \sigma(i), \\
M_{\alpha(i) i}^{\prime} \neq 0, & M_{i j}^{\prime}=0 \quad \forall j \neq \sigma^{-1}(i),
\end{aligned}
$$

and

$$
L_{i \sigma(i)}^{\prime} M_{\sigma(i) i}^{\prime}=g_{i} h_{i}^{T}, \quad M_{\sigma(i) i}^{\prime} L_{\sigma(i) i}^{\prime}=p_{\sigma(i)} q_{\sigma(i)}^{T}
$$

for some $\sigma \in S_{r}$.

Thus $\operatorname{rank} L^{\prime} \geqslant r$. But since $r=\operatorname{rank} L=\operatorname{rank} L^{\prime}+\operatorname{rank} L^{\prime \prime}$, we get, $L^{\prime \prime}=0$ and rank $L^{\prime}=r$. Thus each $L_{i \sigma(i)}^{\prime}$ is of rank 1 and so $L_{i \sigma(i)}^{\prime}$ is of the form $x_{i} y_{\sigma(i)}^{T}$, where $x_{i}>0, y_{\sigma(i)}>0$ and are of sizes $a_{i}, \alpha_{\sigma(i)}$ respectively. We have

$$
A=P_{1}^{T} L Q_{1}=P_{1}^{T}\left[\begin{array}{ll}
L^{\prime} & 0 \\
0 & 0
\end{array}\right] Q_{1} \text {. }
$$

Writing $\sigma$ as a product of disjoint cycles we interchange rows and columns consisting of block entries of $L$ and obtain permutation matrices $P_{2}, Q_{2}$ of suitable orders such that $P_{2} L^{\prime} Q_{2}^{T}$ is a direct sum of matrices of types (a), (b) in the statement of the theorem. Then setting

$$
P=\left[\begin{array}{ll}
P_{2} & 0 \\
0 & I
\end{array}\right] P_{1} \text { and } Q=\left[\begin{array}{ll}
Q_{2} & 0 \\
0 & I
\end{array}\right] Q_{1},
$$

we obtain $P A Q^{T}$ is a direct sum of matrices of types (a), (b), (c) as desired. Since $M_{\sigma(i) i}^{\prime} \neq 0, M_{i j}^{\prime}=0 \forall j \neq \sigma^{-1}(i)$, we, similarly, get $Q W(A W)^{\#} p^{T}$ is a direct sum of matrices of types $\left(\mathrm{a}^{\prime}\right),\left(\mathrm{b}^{\prime}\right),\left(\mathrm{c}^{\prime}\right)$ such that if $S_{i}$ is a summand of $P A Q^{T}$ of type ( ) of order $\lambda_{i} \times \mu_{i}$, then $S_{i}$ is a summand of type (') of order $\mu_{i} \times \lambda_{i}$, completing the proof of the "if" part. The converse is obvious. Further, if $m=n$, the proof shows that $P=Q$.

Before we give some of the consequences of the above theorem we record another simple fact in the following lemma.

Sublemma 3. Let $A$ be an $m \times n$ matrix (not necessarily nonnegative). If $X$ is $a$ $\{1,2\}$-inverse of $A$ such that both $A X$ and $X A$ are weakly 0 -symmetric, then $X$ has the property: the ith row (column) of $X$ is nonzero if and only if the ith column (row) of $A$ is nonzero.

COROllary. Let $A$ be a nonnegaitve $m \times n$ matrix and let $X$ be an $n \times m$ matrix satisfying (1) $A X A=A$, (2) $X A X=X,(3)_{0}(A X)$ is weakly 0-symmetric, $(4)_{0}(X A)$ is weakly 0 -symmetric. Then $X \geqslant 0$ if and only if there exists permutation matrices $P, Q$ of orders $m, n$ such that $P A Q^{T}\left(Q X P^{T}\right)$ is the direct sum of matrices of types (a), (b), $(\mathrm{c}),\left(\left(\mathrm{a}^{\prime}\right),\left(\mathrm{b}^{\prime}\right),\left(\mathrm{c}^{\prime}\right)\right)$ with the convention that if $P A Q^{T}$ has its ith summand of type $(\mathrm{a}),(\mathrm{b})$ 
or (c) of order $\lambda \times \mu$ then $Q X P^{T}$ has its ith summand of type $\left(\mathrm{a}^{\prime}\right),\left(\mathrm{b}^{\prime}\right),\left(\mathrm{c}^{\prime}\right)$ respectively of order $\mu \times \lambda$.

Further, $X=A^{\dagger}$ if and only if $X=D A^{T}=A^{T} D^{\prime}$ where $D, D^{\prime}$ are diagonal matrices such that all entries on each diagonal are positive.

Proof. Firstly, we have $\operatorname{rank} A=\operatorname{rank} A X$ and so, by the Sublemma 3, we put $W=X$ in the theorem. Then $W(A W)^{\#}=X(A X)^{\#}=X$. Thus by Theorem 1 , $P A Q^{T}$ and $Q X P^{T}$ are direct sum of matrices of desired types. The converse is clear. To prove the last statement let us first assume $X=A^{\dagger}$. Then $A X$ and hence $\left(P A Q^{T}\right)\left(Q X P^{T}\right)$ is a symmetric idempotent. Thus if $S_{i}$ and $S_{i}^{\prime}$ are, respectively, the corresponding summands of $P A Q^{T}$ and $Q X P^{T}$ in the direct sum representations described above, then $S_{i} S_{i}^{\prime}$ is also a symmetric idempotent. We now show $S_{i}^{\prime}=D_{i} S_{i}$ $=S_{i} D_{i}^{\prime}$, where $D_{i}, D_{i}^{\prime}$ are diagonal matrices with positive entries on the diagonal. If $S_{i}=0$ and $S_{i}^{\prime}=0$ then $D_{i}$ and $D_{i}^{\prime}$ can be chosen to be the identity matrices of appropriate orders. We now assume that $S_{i}, S_{i}^{\prime}$ are summands of type (b) and (b') respectively. (For summands of types (a) and ( $\left.a^{\prime}\right)$, the proof is similar.) Then

$$
S_{i} S_{i}^{\prime}=\left[\begin{array}{ccc}
x_{1} y_{2}^{T} u_{1} v_{2}^{T} & & 0 \\
& \ddots & \\
0 & & x_{d} y_{1}^{T} u_{d} v_{1}^{T}
\end{array}\right] .
$$

Since $S_{i} S_{i}^{\prime}$ is symmetric, for all $j=1, \ldots, d-1$, we have $x_{j}\left(y_{j+1}^{T} u_{j}\right) v_{j+1}^{T}=$ $v_{j+1}\left(u_{j}^{T} y_{j+1}\right) x_{j}^{T}$ and $x_{d} y_{1}^{T} u_{d} v_{1}^{T}=v_{1} u_{d}^{T} y_{1} x_{d}^{T}$. Thus $v_{j+1}=\alpha_{j} x_{j}, \alpha_{j}>0,1 \leqslant j \leqslant d-1$, and $v_{1}=\alpha_{1} x_{d}, \alpha_{1}>0$. Similarly, by using $S_{i}^{\prime} S_{i}$ is symmetric, we obtain $u_{j}=\beta_{j} y_{j+1}$, $1 \leqslant j \leqslant d-1$, and $u_{d}=\beta_{d} y_{1}, \beta_{d}>0$. Hence $u_{j} v_{j+1}^{T}=\gamma_{j} y_{j+1} x_{j}^{T}, \gamma_{j}>0,1 \leqslant j \leqslant d$ $-1, u_{d} v_{1}^{T}=\gamma_{d} y_{1} x_{d}^{T}, \gamma_{d}>0$. Therefore, $S_{i}^{\prime}=D_{i} S_{i}^{T}=S_{1}^{T} D_{1}^{\prime}$ where $D_{i}, D_{i}^{\prime}$ are diagonal matrices with positive entries on the diagonal. Set

$$
D^{*}=\left[\begin{array}{ccc}
D_{1} & & 0 \\
& D_{2} & \\
0 & & \ddots
\end{array}\right] .
$$

Then $Q A^{\dagger} P^{T}=D^{*}\left(P A Q^{T}\right)^{T}=D^{*} Q A^{T} P^{T}$. This implies $A^{\dagger}=D A^{T}$, where $D=$ $Q^{T} D^{*} Q$ is a diagonal matrix with all entries on the diagonal as positive. Similarly, $A^{\dagger}=A^{T} D^{\prime}$ where $D^{\prime}$ is a diagonal matrix will all entries on the diagonal positive, proving the 'only if' part. To prove the converse, we assume $X=D A^{T}=A^{T} D^{\prime}$. To show $X=A^{\dagger}$, consider $A X=A D A^{T}$ and $X A=A^{T} D^{\prime} A$. Clearly. both $A X, X A$ are symmetric. Hence $X=A^{\dagger}$ as desired. This completes the proof of the corollary.

REMARK. The last statement in the above corollary, that if $A \geqslant 0$ and $A^{\dagger} \geqslant 0$ then $A^{\dagger}=D A^{T}$, is a theorem of Plemmons-Cline [2]. Henceforth, by matrices of types (a), (b), (c), we shall mean the matrices of such types described in Theorem 1.

THEOREM 2. Let $A$ be a nonnegative $m \times n$ matrix and $W$ be a nonnegative $n \times m$ matrix such that the ith row (column) of $W$ is nonzero if and only if the ith column (row) of $A$ is nonzero. Let $X$ be $W$-weighted group inverse of $A$. Then $X \geqslant 0$ iff $P X Q^{T}$ is a direct sum of matrices of types (a), (b) and (c). 
Proof (if part). It is immediate from the equations $(1)_{w}(A W)^{2} X W=A W(2)_{w}$ $X=X W A W X$ and (5) $)_{w} X W A=A W X$ that $\operatorname{rank} X=\operatorname{rank} X W=\operatorname{rank} A W=$ rank $W A W$. Also $X W=(A W)^{\#}, W X=(W A)^{\#}$. Thus $X W A W$ and $W A W X$ are nonnegative idempotents which are also weakly 0-symmetric (Lemma 1).

Thus there exist permutation matrices $P, Q$ such that

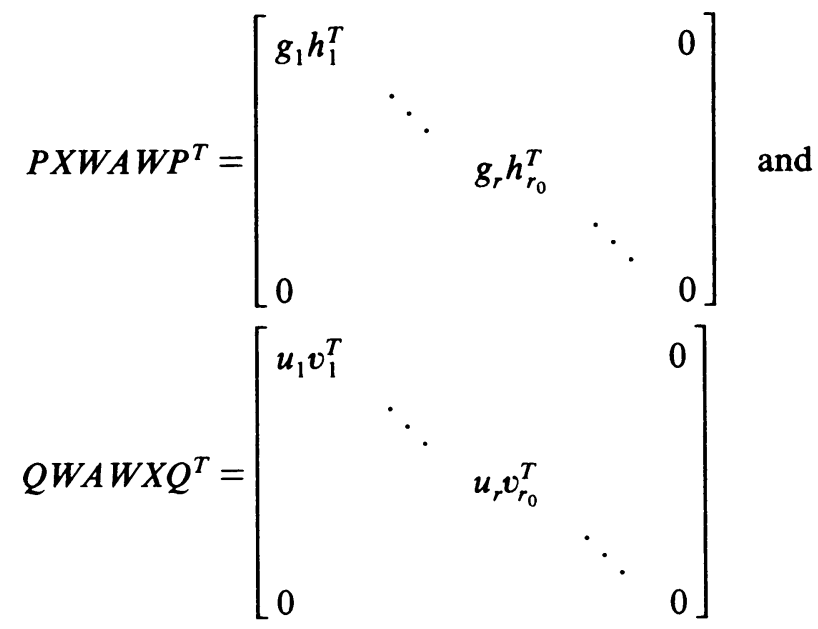

where $r=\operatorname{rank} X=\operatorname{rank} W A W$. Then invoking Lemmas 2 and 3 we obtain, as in the proof of Theorem 1, that $P X Q^{T}$ is a direct sum of matrices of types (a), (b) and (c) as desired. The converse is obvious.

Remark (1). Let $W=A^{\dagger}$. Then since $X=\left((A W)^{\#}\right)^{2} A$ [3], we obtain $X=A$. Thus a nonnegative matrix $A$ with $A^{\dagger} \geqslant 0$ is a direct sum of matrices of the types (a), (b), and (c), up to permutations of rows and columns. Stated equivalently, we obtain a theorem of Plemmons-Cline [2] that if $A \geqslant 0$ and $A^{\dagger} \geqslant 0$ then there exists a permutation matrix $P$ such that

$$
P A=\left[\begin{array}{l}
B_{1} \\
\vdots \\
B_{\gamma} \\
0
\end{array}\right],
$$

where each $B_{i}$ has rank $1, B_{i} B_{j}^{T}=0$ for $i \neq j$ and the zero block may be absent.

REMARK (2). We can also choose $W=\{1,3,4\}$-inverse of $A$. In this case we obtain the same class of matrices $A$ as in the case when we choose $W=A^{\dagger}$ which is a result of Berman-Plemmons [1]. However, we cannot choose $W$ to be any other $\lambda$-inverse of $A$ where $\lambda$ is a subset of $\{1,2,3,4\}$ such that $1 \in \lambda$. For, if $W$ is any other $\lambda$-inverse of $A$ such that $1 \in \lambda$ then, although $\operatorname{rank} A W=\operatorname{rank} A, X=\left((A W)^{\#}\right)^{2} A=A W A$ $=A$, it is not necessarily true that the $i$ th row (column) of $W$ is nonzero if and only if the $i$ th column (row) of $A$ is nonzero. This suggests that one may weaken the hypothesis on $W$ regarding nonzero rows and nonzero columns to obtain other results on $\lambda$-monotone matrices. This requires finding matrices $X, Y$ such that $X Y$ and $Y X$ are not necessarily in the form as in Lemma 2 . This remains open. 
ACKNOWLEDGement. I gratefully acknowledge the invitation of Professor Robert L. Plemmons in February 1979 to the University of Tennessee and the stimulating conversations with him and Professor Randy E. Cline. It was during this visit that Professor Cline proposed the problem studied in this paper.

\section{REFERENCES}

1. A. Berman and R. J. Plemmons, Inverses of nonnegative matrices, Linear and Multilinear Algebra 2 (1974), 161-172.

2. R. J. Plemmons and R. E. Cline, The generalized inverse of a nonnegative matrix, Proc. Amer. Math. Soc. 31 (1972), 46-50.

3. R. E. Cline and T. N. E. Greville, A Drazin inverse for rectangular matrices, Linear Algebra Appl. 29 (1980), 53-62.

4. P. Flor, On groups of nonnegative matrices, Compositio Math. 21 (1969), 376-382.

5. S. K. Jain, V. K. Goel and Edward K. Kwak, Nonnegative mth roots of nonnegative 0-symmetric idempotent matrices, Linear Algebra Appl. 23 (1979), 37-52.

6. ___ Nonnegative matrices having some nonnegative Moore-Penrose and group inverses, Linear and Multilinear Algebra 7 (1979), 59-72.

7. ___ Decomposition of nonnegative group-monotone matrices, Trans. Amer. Math. Soc. 257 (1980), $371-385$.

8. S. K. Jain and L. E. Snyder, Nonnegative $\lambda$-monotone matrices, SIAM J. Algebraic Discrete Methods 2 (1981), 66-76.

Department of Mathematics, Ohio University, Athens, Ohio 45701 